\title{
Systems Biology of Mammalian Circadian Clocks
}

\author{
H.R. UEDA \\ Laboratory for Systems Biology and Functional Genomics Unit, \\ Center for Developmental Biology, Riken, Kobe, Hyogo 650-0047, Japan
}

\begin{abstract}
Systems Biology is a natural extension of molecular biology and can be defined as biology after identification of key gene(s). Systems-biological research is hence seen as a multistage process, beginning with the comprehensive identification and quantitative analysis of individual system components and their networked interactions and leading to the ability to control existing systems toward the desired state and design new ones based on an understanding of structure and underlying dynamical principles. In this chapter, we take mammalian circadian clocks as a model system and describe systems-biological approaches, including the identification of clock-controlled genes, clock-controlled cis elements, and clock transcriptional circuits driven by functional genomics; the parameter change of clock components followed by quantitative measurement; and the dynamic and quantitative perturbation of the clock and its application to one of the fundamental but yet-unsolved questions: singularity behavior of clocks. As perspective for systems-biological investigations, we also introduce the system-level dynamical questions related to the core of clocks, including delay, nonlinearity, temperature-compensation and synchronization of mammalian circadian oscillator(s), and the system-level information problems related to clocks in the environment, including the internal representation of light change through perfect adaptation and internal representation of day length through photoperiodism in mammals.
\end{abstract}

\section{INTRODUCTION}

\section{Systems Biology as "Biology after Identification"}

Recent large-scale efforts in genome sequencing, expression profiling, and functional screening have produced an embarrassment of riches for life science researchers, and biological data can now be accessed in quantities that are orders of magnitude greater than were available even a few years ago. The growing need for interpretation of data sets, as well as the accelerating demand for their integration to a higher-level understanding of life, has set the stage for the advent of systems biology (Kitano 2002a,b), in which biological processes and phenomena are approached as complex and dynamic systems. Systems biology is a natural extension of molecular biology and can be defined as "biology after identification of key gene(s)." We see systems-biological research as a multistage process, beginning with the comprehensive identification and quantitative analysis of individual system components and their networked interactions and leading to the ability to control existing systems toward the desired state and design new ones based on an understanding of structure and underlying dynamical principles (Fig. 1)

\section{Mammalian Circadian Clock as a Model System}

We have taken the mammalian circadian clock as an initial model system that exhibits system-level dynamical and structural properties in order to develop research strategies and technologies for studies of complex and dynamic biological systems. The mammalian circadian clock consists of complexly integrated feedback and feedforward loops (Reppert and Weaver 2002) and also exhibits well-defined dynamical properties (Dunlap et al. 2004), including (1) endogenous oscillations of an approximately 24-hour period, (2) entrainment to external environmental changes (temperature and light cycle), (3) temperature-compensation over a wide range of temperature, and (4) synchronization of multiple cellular clocks despite the inevitable molecular noise. All of these dynamical properties would be difficult to elucidate without utilizing such system-level approaches. In addition to its advantage as a basic model system for systems-biological research, the function of the circadian clock is intimately involved in the control of metabolic and physiological processes (Panda et al. 2002; Reppert and Weaver 2002), and its dysregulation is associated with the onset and development of numerous human diseases, including sleep disorders, depression, and dementia. An improved understanding at the systems level promises to provide biomedical and clinical investigators with a powerful new arsenal for attacking these conditions.

\section{Development of Systems-Biological Approaches and Their Application to Clocks}

Attempts to elucidate the design principles of complex and dynamic biological systems such as the mammalian circadian clock may require (1) identification of wholenetwork structure through comprehensive (genome-wide) screening (system identification), (2) prediction and validation to derive the design principle through the accurate measurement of network behaviors (system analysis), (3) repair and control of network state toward the desired state through the precise perturbation of its components (system control) and ultimately, (4) reconstruction and design of new systems based on the design principles derived from the identified structure and observed dynamics of the original network (system design). To develop these systems-biological approaches, we have focused mainly on the development and evaluation of 


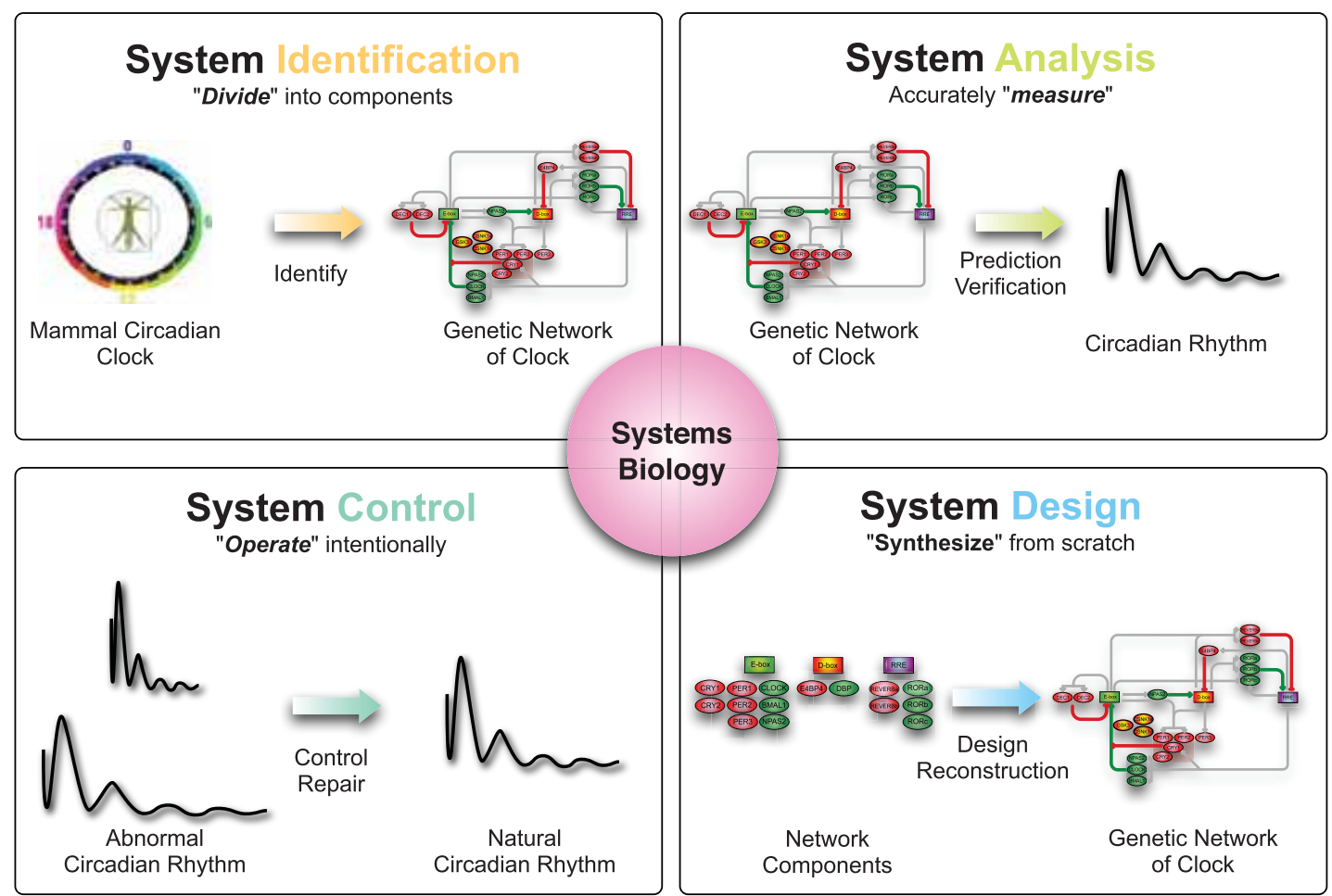

Figure 1. Systems biology. Systems-biological research starts with comprehensive identification (upper left panel). In this step, individual system components and their networked interactions are comprehensively identified. In the second step, to derive the design principle of a target system, the behavior of the system is predicted and validated through an accurate measurement with perturbations (upper right panel). An understanding of the design principle of the system is essential to derive the method of controlling the system toward the desired state (lower left panel). Finally, the level of understanding is confirmed by reconstruction of the system (lower right panel).

technologies and strategies. For each of these processes, we have been able to report several strategies and technologies as well as their application to specific questions in mammalian circadian clocks. This has included the identification of clock-controlled genes, clock-controlled cis elements, and clock transcriptional circuits driven by functional genomics (Ueda et al. 2002c, 2005) in system identification, parameter change of clock components by quantitative measurement (Sato et al. 2006) in system analysis, dynamic and quantitative perturbation of clock and its application to one of the fundamental but yetunsolved questions, and singularity behavior of clocks (Ukai et al. 2007) in system control. As for the effort of system design, we recently initiated our current project on in cellulo and in vitro reconstruction of mammalian circadian circuits. In the following sections, we describe in detail these strategies and/or technologies developed for each stage of systems-biological research, in addition to their application to the specific question of mammalian circadian clocks.

\section{SYSTEMS-BIOLOGICAL APPROACHES}

\section{Identification of Clocks}

Following the completion of genome projects for species such as mouse and human, genome-wide resources such as small interfering RNA (siRNA) or cDNA libraries have undergone considerable expansion.
Development of high-throughput technologies has also assisted in the efficient use of these resources. These genome-wide resources and technologies and genomeassociated information currently allow us to comprehensively identify system components of interest (system identification).

Circadian clocks of multicellular organisms consist of complex integrated regulatory loops with positive or negative regulators known as clock genes (Wuarin and Schibler 1990; King et al. 1997; Zylka et al. 1998; van der Horst et al. 1999; Vitaterna et al. 1999; Bunger et al. 2000; Lowrey et al. 2000; Bae et al. 2001; Mitsui et al. 2001; Reick et al. 2001; Zheng et al. 2001; Honma et al. 2002; Preitner et al. 2002; Sato et al. 2004). The transcriptional regulation network of these genes forms a circadian clock oscillator, which is known to control output genes and to affect physiological and metabolic processes (Panda et al. 2002; Reppert and Weaver 2002). Although some transcriptional regulations of identified clock genes have been the subject of previous studies, a system-level understanding of circadian clocks remains to be elucidated. In this section, we provide results of our system identification of mammalian circadian clocks (Ueda et al. 2002c, 2005).

Identification of the mammalian clock circuit. The mammalian circadian master clock is primarily located in the suprachiasmatic nucleus (SCN) (Reppert and Weaver 2002). Transcript analyses have indicated that 
circadian clocks are not restricted to the SCN but are found in several tissues (Yamazaki et al. 2000) including liver and cultured fibroblast cells such as Rat-1 (Balsalobre et al. 1998; Yagita et al. 2001) or NIH-3T3 (Akashi and Nishida 2000) cells. The mechanisms underlying circadian rhythms are also known to be conserved across species (Dunlap et al. 2004). At the basic core of the clock lies a transcriptional/translational feedback loop (Gekakis et al. 1998; Kume et al. 1999; Shearman et al. 2000), whose primary components are known as "clock genes" (Wuarin and Schibler 1990; King et al. 1997; Zylka et al. 1998; van der Horst et al. 1999; Vitaterna et al. 1999; Bunger et al. 2000; Lowrey et al. 2000; Bae et al. 2001; Mitsui et al. 2001; Reick et al. 2001; Zheng et al. 2001; Honma et al. 2002; Preitner et al. 2002; Sato et al. 2004). For example, in the mouse system, the transcription factors CLOCK and BMAL1 proteins dimerize and directly and indirectly activate transcription of the Per and Cry genes through E-box elements (5'-CACGTG-3') (Gekakis et al. 1998; Kume et al. 1999). The PER and CRY proteins accumulate in the cytosol and are then translocated following phosphorylation into the nucleus where they inhibit the activity of CLOCK and BMAL1 (Reppert and Weaver 2002). The turnover of the inhibitory PER and CRY proteins leads to a new cycle of activation by CLOCK and BMAL1 via E-box elements. Despite the reporting of many transcriptional regulations of each gene, however, an overview of the circadian clock core network remains to be put forward.

Complicated networks cannot be elucidated without access to both (1) comprehensive identification of network circuits and (2) accurate measurement of system dynamics. In a previous attempt to comprehensively identify the circadian clock core network, we first quantitatively and comprehensively measured genome-wide gene expression using a high-density oligonucleotide probe array (Lipshutz et al. 1999) and identified genes showing circadian oscillation with characteristic expression patterns through biostatistics (Fig. 2). The second step involved comprehensively determining the transcription start sites (TSSs) (Suzuki et al. 2001) and conserved noncoding regions to construct a genomewide promoter/ enhancer database. Using these data, we predicted that there was a relationship between expression patterns of identified genes and DNA regulatory elements on their promoter/enhancer regions. We found that clock-controlled elements (CCEs), E boxes (5'CACGTG-3'; Hogenesch et al. 1998), E' boxes (5'CACGTT-3'; Ueda et al. 2005; Yoo et al. 2005), D boxes (5' -TTATG[C/T]AA-3'; Falvey et al. 1996), or Rev-response elements (RREs) (5'-[A/T]A[A/T]NT [A/G]GGTCA-3'; Harding and Lazar 1993) are distributed throughout the oscillatory genes.

To determine the role of these elements in the circadian clock, we utilized a cell culture system with which we can monitor circadian rhythms in transcriptional dynamics using a destabilized luciferase $(\mathrm{d} L u c)$ reporter driven by clock-controlled promoters (Fig. 3A). In this cell culture system-named "in cellulo cycling assay"-we transiently transfected reporter constructs into cultured Rat-1 cells and stimulated them with dexamethasone and measured their bioluminescences. Dexamethasone was administrated to induce macroscopic circadian oscillations in the cultured cells. Through the genome-wide searching described above, we found CCEs on 16 clock/clock-controlled gene promoter/enhancers. Then, using the in cellulo cycling assay system, we were able to reveal that functionally and evolutionary conserved $\mathrm{E} / \mathrm{E}^{\prime}$ boxes are located on noncoding regions of nine genes (Per1, Per2,

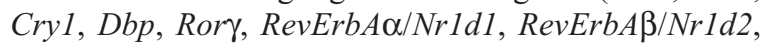
Dec1/Bhlhb2, and Dec2/Bhlhb3), D boxes on those of seven genes (Per1, Per 2, Per3, RevErbA $\alpha$, RevErbA $\beta$, Ror $\alpha$, and Ror $\beta$ ), and RREs on those of six genes (Bmall/Arntl, Clock, Npas2, Cry1, E4bp4/Nfil3, and Ror $\gamma)$. On the basis of this functional and conserved transcriptional regulatory mechanism, we succeeded in drawing transcriptional circuits underlying mammalian circadian rhythms (Fig. 3B) (Ueda et al. 2005).

Our analysis further suggested that regulation of $\mathrm{E} / \mathrm{E}^{\prime}$ boxes is the topological vulnerability point in mammalian circadian clocks. We functionally verified this concept using in cellulo cycling assay system (Fig. 3C). Overexpression of repressors of $\mathrm{E} / \mathrm{E}^{\prime}$ box regulation (CRY1; Kume et al. 1999), RRE regulation (REVERBA $\alpha$; Preitner et al. 2002; Ueda et al. 2002c), or D-box regulation (E4BP4; Mitsui et al. 2001) affected circadian rhythmicity in Per2 or Bmall promoter activity. The effects were different, however, between each repressor, and the severest effect was observed when the E/E' box was attacked. Such different modes of effects cannot be explained by mere quantitative differences in the strength of these three repressors, indicating that there is some qualitative difference between $\mathrm{E} / \mathrm{E}^{\prime}$ box, D-box, and RRE regulation in circadian rhythmicity (Ueda et al. 2005).

\section{Analysis of Clocks}

To derive the design principles of a system of interest, it is important to validate the behavior of a predicted system through an accurate measurement with several types of perturbations (system analysis). In our efforts to identify the circadian clock system, we have succeeded in drawing the circadian transcriptional circuits and revealed the topological importance of the morning element, $\mathrm{E}$ box. In this section, we describe our effort to validate the hypothesis that transcriptional feedback repression through the E box is required for mammalian clock function. In this study, we collaborated with Dr. John B. Hogenesch's and Dr. Steve A. Kay's groups to change the molecular parameter for feedback repression by functional genomics and then tested the cellular phenotype caused by this parameter change by our in cellulo cycling assay system. Through this study, we have demonstrated the necessity of transcriptional repression for circadian clock function (Sato et al. 2006).

Negative feedback as the heart of the transcriptional circuit in mammalian clocks. Circadian clocks have been proposed as consisting of autoregulatory loops in which transcriptional feedback and regulated protein turnover are used to maintain a 24-hour periodicity (Dunlap 1999; 
A

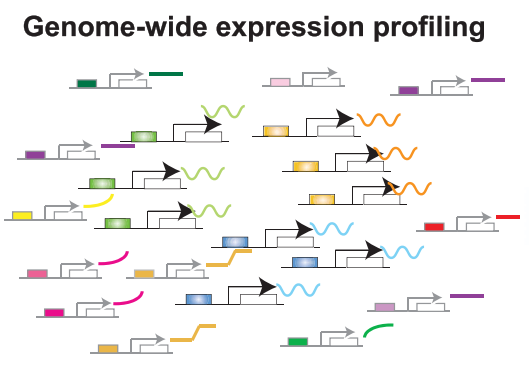

B

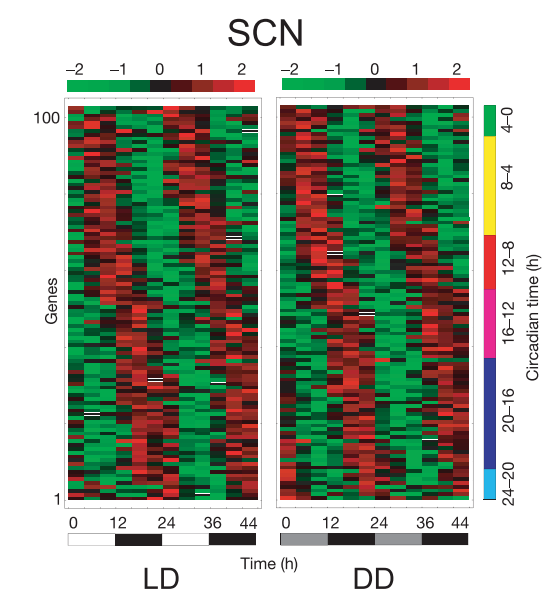

Promoter analysis of co-expressed genes
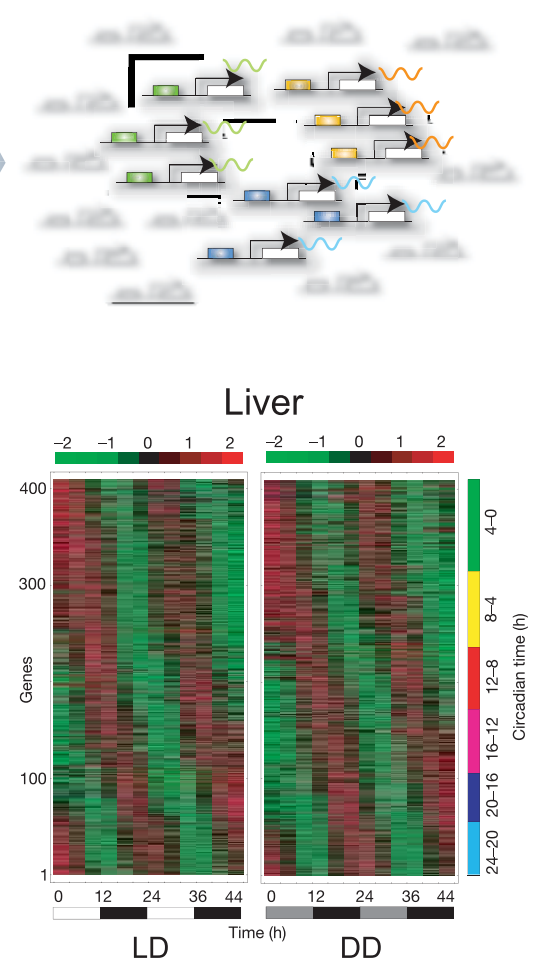

C

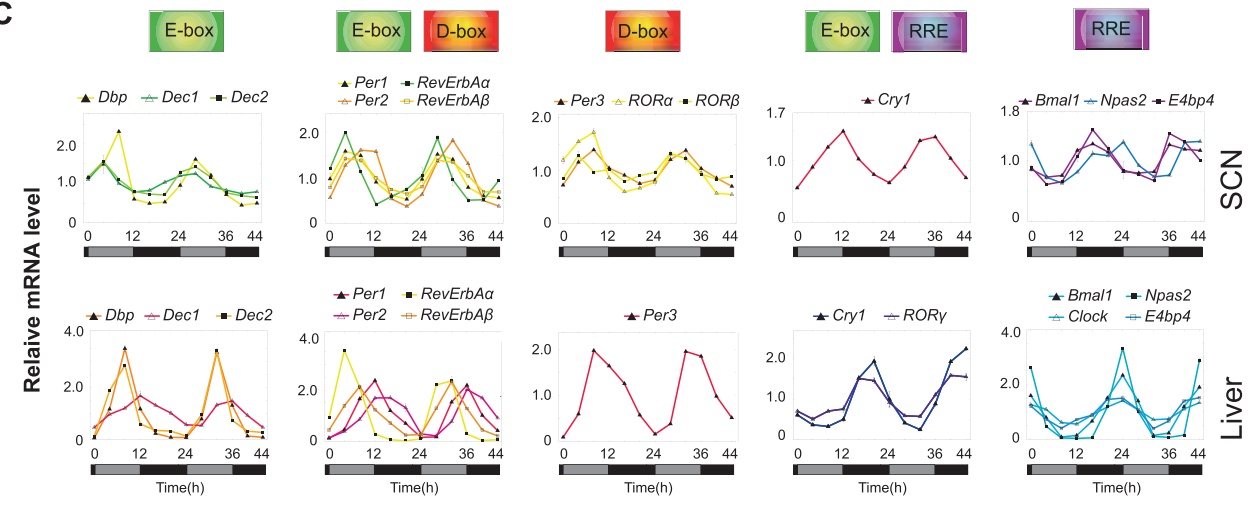

Figure 2. (A) Strategy for identification of clock-controlled elements (CCEs). Gene expression information was obtained by performing comprehensive expression profiling (left panel). Through statistical analysis, genes with a special characteristic pattern of expression (circadian oscillation) were selected (right panel, oscillatory genes are indicated by their color). DNA regulatory elements for specific issue (i.e., expression timing) were predicted by combining expression pattern information and transcriptional regulatory element information from promoter regions. $(B)$ Genome-wide expression profiles in mouse central (SCN, left panel) and peripheral (liver, right panel) clocks. Total RNA were extracted every 4 hours during light/dark cycles (LD) or constant darkness (DD) over 2 days and used to determine genome-wide gene expression profiles with an Affymetrix mouse high-density oligonucleotide probe array. Data were normalized so that the average signal intensity and standard deviation over 12-point time courses were 0.0 and 1.0, respectively. Columns represent time points, and rows represent genes that were organized by peak time. Colors in descending order from red to black to green represent the normalized data. From the obtained data, we identified a set of genes rhythmically expressed under both LD and DD. We classified 101 genes in the SCN and 393 genes in the liver as "significantly rhythmic under both LD and DD." $(C)$ Temporal expression profiles of transcription factors in the SCN (upper panel) and liver (lower panel) under DD conditions. Relative mRNA levels under DD conditions of the indicated genes were measured with quantitative polymerase chain reaction (Q-PCR) assay, in which GAPDH expression was used as an internal control. Data were normalized so that the average copy number (Q-PCR) over a 12-point time course was 1.0. Circadian expression of transcription factors having functional and evolutionary conserved E boxes (Dbp, Dec1, and Dec2), both E boxes/E' boxes and D boxes (Perl, Per2, RevErbA $\alpha$, and RevErbA $\beta$ ), D boxes (Per3, Ror $\alpha$, and Ror $\beta$ ), RREs (Bmall, Clock, Npas 2, and E4bp4), both E boxes/E' boxes and RREs (Cryl and Ror $\gamma$ ) on their noncoding regions. Clock and Ror $\gamma$ were constitutively expressed in the SCN. (Modified from Ueda et al. 2002c, 2005.) 
A

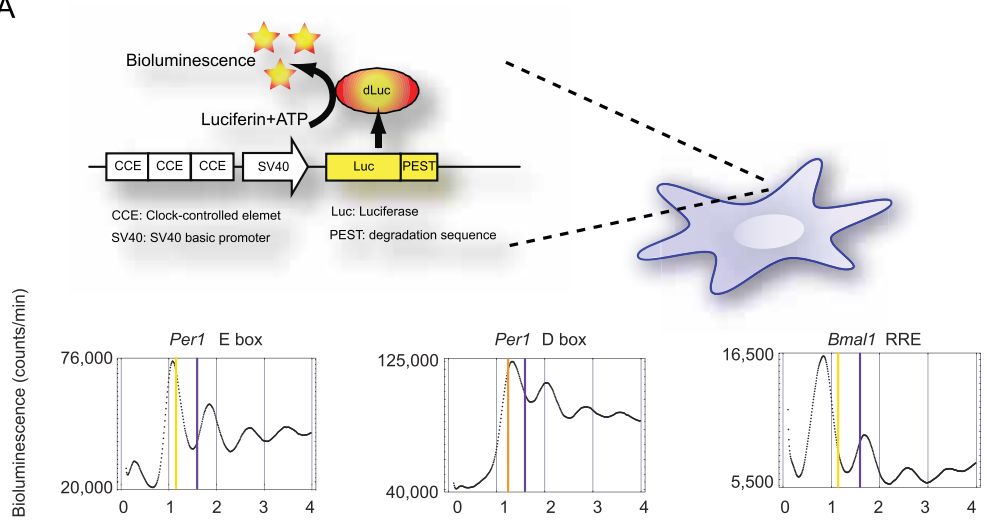

B

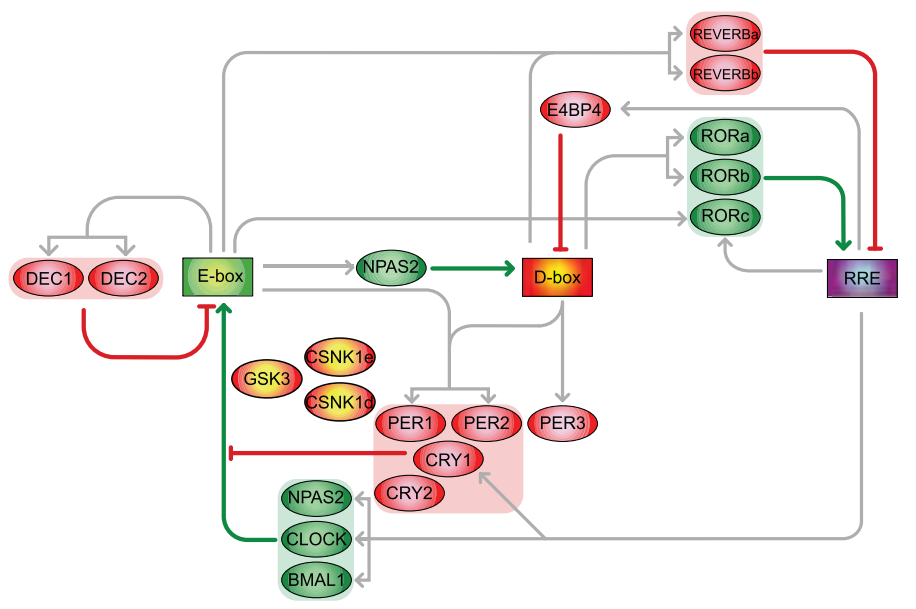

C
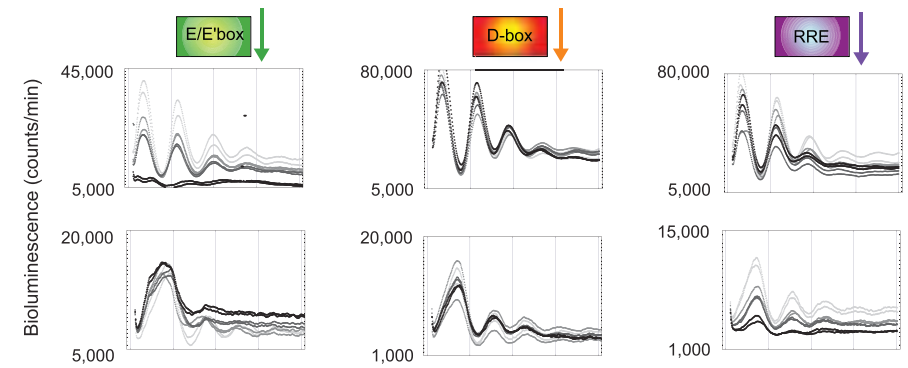

Figure 3. (A) Schematic overview of the experiment. Cultured mammalian cells (Rat-1) were transfected with $\mathrm{d} L u c$ under the regulation of CCE and SV40 basic promoter. The circadian change of bioluminescence was monitored by a PMT detector over several days (upper panel). Representative circadian rhythms of bioluminescence from wild-type CCE fused to the SV40 basic promoter driving a $\mathrm{d} L u c$ reporter. The circadian bioluminescence phase from the Per 2 promoter and that of the Bmall promoter are marked by yellow and purple lines, respectively (bottom panels). (B) Schematic representation of the transcriptional network of the mammalian circadian clock. (Ellipsoids) Genes; (rectangles) CCEs. Transcriptional-translational activation and repression are depicted as gray, green, and red lines, respectively. (C) Effect of repression on each CCEs. The E/E' boxes, D box, and RRE are repressed by overproduction of CRY1, E4BP4, and REVERBA $\alpha$, respectively. The consequences of those repressions were monitored by Per2-dLuc (upper panel) and Bmall-dLuc (lower panel). (dLuc) Destabilized luciferase; (CCE) clock-controlled elements; (PMT) photomultiplier tube. (Modified from Ueda et al. 2005.)

Young and Kay 2001; Reppert and Weaver 2002). The universal necessity for transcriptional feedback repression, however, arose as a question mainly from recent studies about the cyanobacterial circadian rhythms in which repression was shown not to be necessary (Nakajima et al. 2005; Tomita et al. 2005). Circadian feedback repression in mammals is believed to be mediated by CRYPTOCHROME (CRY1, CRY2) (Kume et al. 1999; van der Horst et al. 1999; Vitaterna et al. 1999) and PERIOD (PER1 and PER2) (Tei et al. 1997; Zheng et al. 1999, 2001) proteins. CRY and PER proteins are hypothesized to autoregulate their own expression by repressing 
the heterodimeric complex of the basic helix-loop-helix (bHLH) PERARNT-SIM (PAS) domain transcriptional activators CLOCK and BMAL1, which bind to E-box elements in the CRY (Etchegaray et al. 2003) and PER (Gekakis et al. 1998; Ueda et al. 2005; Yoo et al. 2005) promoters. However, direct evidence for the requirement of CRY-mediated repression of CLOCK/BMAL1 transcriptional activity in the maintenance of circadian clock function has yet to be presented. Here, in a fruitful collaboration with the J.B. Hogenesch and the S.A. Kay groups, we have successfully shown that feedback repression is actually required for mammalian circadian clock function (Sato et al. 2006).

To determine the requirement of feedback repression in circadian clock function, we sought to identify the CLOCK alleles that were insensitive to CRY1 repression but maintained normal transcriptional activity. Our collaborative partners first generated a library of approximately 6000 random point mutations of human alleles for both CLOCK and BMAL1 and then screened clones individually in cell-based reporter assays with wild-type Bmall cDNA and a Perl promoter-luciferase (Perl-Luc) construct (Gekakis et al. 1998) in the presence of cotransfected Cryl. Of the CLOCK and BMAL1 clones screened, several reproducibly maintained threefold or greater reporter activity in the presence of CRY1 compared with wild-type alleles. Notably, these clones demonstrated transcriptional activities similar to those of wild type in the absence of cotransfected Cryl, suggesting that these mutations do not cause overt alterations in the heterodimerization, nuclear localization, DNA-binding, and trans-activation properties of the mutant CLOCK/BMAL1 complex.

The prevailing transcriptional feedback model (Fig. $4 \mathrm{~A})$ predicts that impairment of CRY-mediated repression should have marked effects on circadian expression of the Per genes. This notion is supported by in vivo observations that expression of Per 1 and Per 2 is constitutively elevated in Cry $1 / \mathrm{Cry} 2$ double-knockout mice (Okamura et al. 1999; Vitaterna et al. 1999). To determine whether these mutations in CLOCK and BMAL1 cause phenotypic changes in circadian gene expression, we performed an in cellulo cycling assay (Ueda et al. 2002b, 2005) (see also above, Identification of Clocks). Mouse NIH-3T3 cells were transfected with plasmids harboring destabilized luciferase $(\mathrm{d} L u c)$ driven by Per 2 or SV40 basic promoters along with the BMAL1 and CLOCK mutant allele and then monitored using the in cellulo cycling assay (Fig. 4B, left). Cotransfection of wild-type CLOCK and BMAL1 did not substantially alter rhythmicity compared with empty vector transfection, as their period lengths were 21.4 hours \pm 0.4 . In contrast, when compared with wild-type CLOCK/BMAL1, transfection of either of CLOCK or BMAL1 mutant alleles resulted in substantial impairment of circadian rhythmicity after one or two cycles of oscillations. Notably, cotransfection of the CRY-insensitive mutant CLOCK and BMAL1 together resulted in the loss of circadian Per2 promoter activity. We were therefore able to demonstrate that the transcriptional repression of CLOCK/BMAL1 by CRY was required for circadian E-box activity.
In addition to Per and Cry, the rhythmic expression of Bmall mRNA is also under circadian clock regulation (Shearman et al. 2000). The Bmall promoter used in this study, however, does not have E-box sites but instead contains RRE (Preitner et al. 2002; Ueda et al. 2002c), whose activities are reciprocally controlled by the rhythmically expressed transcriptional repressor REVERBA $\alpha$ (Preitner et al. 2002) and activator ROR $\alpha$ (Sato et al. 2004). As an additional test for circadian clock function, we examined the effects of mutant CLOCK and BMAL1 on rhythmic RRE activity by in cellulo cycling assays with a Bmall-dLuc reporter. Similar to the results with the Per2-dLuc reporter, transfection of single CLOCK or BMAL1 mutants resulted in the decreased amplitude of cycling of Bmall-dLuc activity compared with wild-type CLOCK/ BMAL1 transfection. Moreover, this decrease in cycling amplitude was further exacerbated upon cotransfection of the double-mutant heterodimer (Fig. $4 \mathrm{~B}$, right). These results indicate that transcriptional repression of CLOCK/BMAL1 by CRY is also required for circadian BMAL1 expression through RRE, which is dependent on the transcriptional, translational, and posttranslational actions of endogenous cellular factors.

Arrhythmic Per2 expression seen from a population of cells expressing the double-mutant CLOCK/BMAL1 complex may be due to the disruption of oscillator function or a lack of synchrony between individual rhythmic cells. To address these possibilities, we measured quantitative imaging of Per2- $\mathrm{d} L u c$ reporter activity from individual NIH-3T3 cells by using an approach similar to that used in analyzing Bmall reporter rhythms from single cells (Welsh et al. 2004). As with the whole-well assays, the median reporter activity for the population of imaged individual cells coexpressing wild-type CLOCK/BMAL1 oscillated rhythmically (Fig. 4C, left). In contrast, the population of individual Clock/Bmall mutant cells (Fig. $4 \mathrm{C}$, right) was visibly arrhythmic. Individual reporter activities from single wild-type cells were rhythmic, as expected, whereas individual Clock/Bmall doublemutant cells showed arrhythmic reporter activities. These differences in activity patterns were evaluated by two independent statistical methods that score the circadian rhythmicity of experimental time-course data.

This is the first study that shows functional necessity for feedback repression in the mammalian circadian clock. Proof of this necessity is important, as recent studies of the cyanobacterial clock found that circadian oscillations in protein phosphorylation can be maintained in the absence of transcriptional feedback repression in vivo (Tomita et al. 2005) and with purified proteins in vitro (Nakajima et al. 2005). We therefore sought to formally test the requirement of CRY-mediated transcriptional feedback repression in mammalian circadian clock function by developing and implementing a new unbiased cellular genetics approach that uses robust mutagenesis techniques and mammalian cell-based screening. Our data presented here provide direct evidence that CRYmediated feedback repression of the CLOCK/BMAL1 complex is required for mammalian clock function. Although it is likely that the mammalian clock is governed by a combination of both transcriptional and non- 


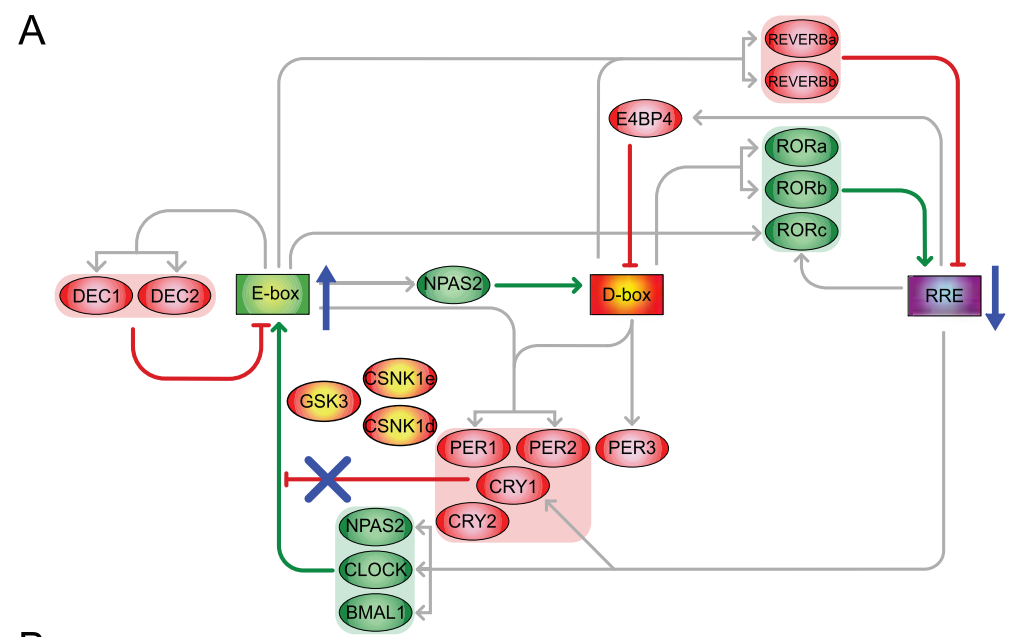

B
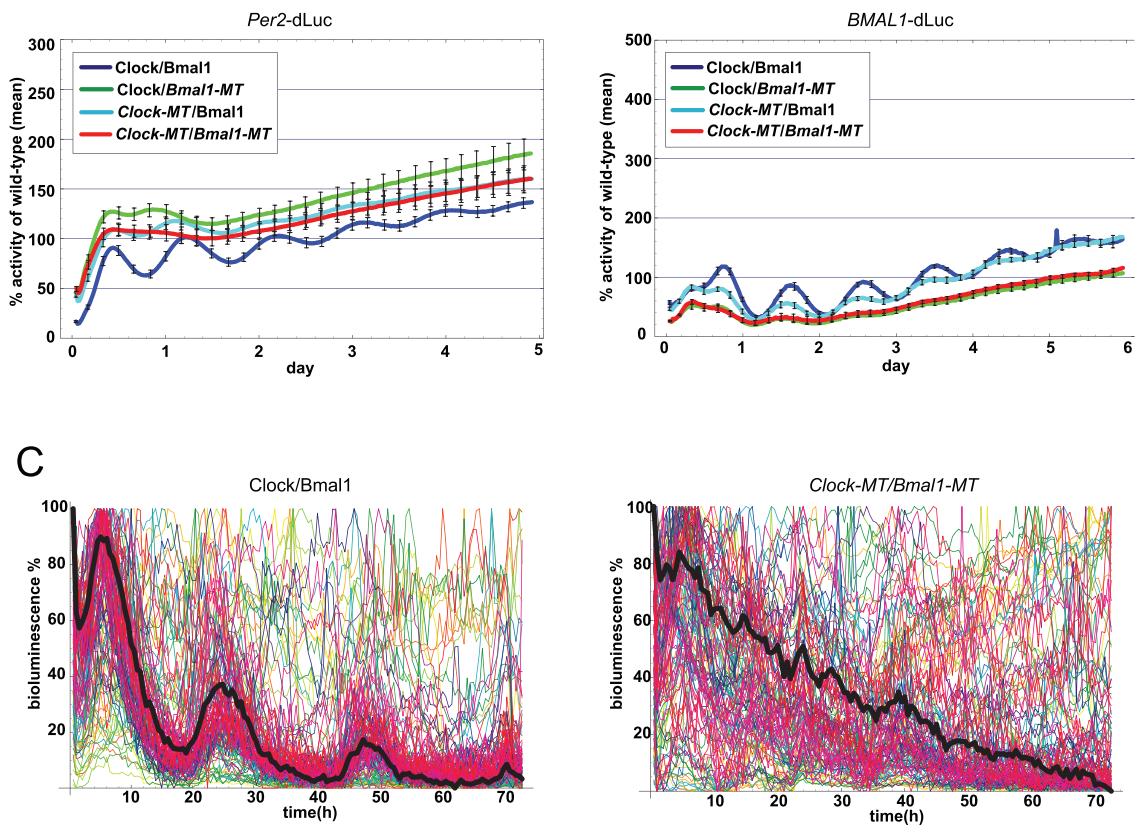

Figure 4. (A) Schematic representation of the transcriptional network of the mammalian circadian clock. (Ellipsoids) Genes; (rectangles) CCE. Transcriptional-translational expression, activation and repression are depicted as gray, green, and red lines, respectively. (B) Coexpression of CLOCK/BMAL1 mutant heterodimers that are insensitive to CRY repression ablates circadian E-box and RRE activities in NIH-3T3 cells. Plasmids expressing Flag-tagged Clock and Bmall alleles were transiently cotransfected with the Per2$\mathrm{d} L u c$ reporter plasmid into NIH-3T3 cells. (Left panel) Per2 promoter activities in NIH-3T3 cells transfected with single or double CRY1-insensitive Clock and Bmall mutants were monitored over 5 days. (Right panel) Bmall promoter activities in NIH-3T3 cells transfected with single or double CRY-insensitive mutants of Clock and Bmall were monitored over 6 days. All reporter activities were normalized such that the median wild-type luciferase activity over the time course was $100 \%$. (C) Coexpression of CLOCK/BMAL1 mutant heterodimers impairs circadian rhythmicity in individual cells. Per2-Luc reporter activities from individual NIH-3T3 cells $(n$ = 133) transfected with Flag-tagged wild-type CLOCK/BMAL1 or double-mutant Clock-MT/Bmall-MT were monitored over 3 days. Reporter activities from each wild-type or double-mutant cells were normalized such that the maximum bioluminescence value was set to $100 \%$ for each panel. The mean reporter activity for all analyzed single cells at each time point is indicated by a black line. (Modified from Sato et al. 2006.)

transcriptional feedback mechanisms, any residual circadian properties that remain upon uncoupling of transcriptional feedback are insufficient to maintain circadian transcriptional output and molecular clock function. Finally, we predict that the application of cellular genetics technology will have a significant impact on mammalian biology as similar approaches have had on prokaryotic and yeast biology.

\section{Control of Clocks}

System control aims to regulate the target system toward the desired state through the precise perturbation of its components. To achieve this, it is necessary to develop an assay system that can be controlled with dynamic and quantitative perturbation.

The circadian clock is known to be entrainable by 
external cues such as light. The light information is transmitted to the circadian clock through sensing mechanisms containing photoreceptors, and as a result of light pulse, the clock system shows a drastic change of its dynamics. In this section, we show the success of control of the oscillating clock system in individual cultured cells via artificial light-sensing mechanisms. We also applied this photoperturbation system to one of the long-standing and unsolved biological phenomena known as the singularity behavior of circadian clocks (Ukai et al. 2007).

Singularity behaviors of circadian clocks. Circadian clocks exhibit various dynamic properties, making them difficult to elucidate without quantitative perturbation and precise measurement of their dynamics. One of the most fundamental but yet-unsolved dynamic properties of circadian clocks is singularity behavior, in which robust circadian rhythmicity can be abolished after a certain critical stimulus, such as light and temperature pulses applied at the appropriate timing and strength. Since the first report of singularity behavior in Drosophila pseudoobscura by Arthur T. Winfree (1970), circadian clock singularities have been experimentally observed in various organisms including unicells such as Gonyaulax (Taylor et al. 1982), Euglena (Malinowski et al. 1985), Chlamydomonas (Johnson and Kondo 1992), fungi (Huang et al. 2006), insects (Winfree 1980), plants (Engelmann et al. 1978; Covington et al. 2001), and mammals (Jewett et al. 1991; Honma and Honma 1999), suggesting that this behavior is a shared property of an extremely broad range of circadian clocks (Dunlap et al. 2004).

Although singularity behavior has been widely observed, little is known about the underlying mechanisms. Since such behaviors were experimentally observed at the multicell level (i.e., the collective behavior of unicells or the physiological or locomoter activity of a multicellular organisms), two alternative single-celllevel mechanisms have been proposed to explain their collapse to singularity: (1) arrhythmicity of individual clocks (Fig. 5A) or (2) desynchronization of individual rhythmically oscillating clocks (Fig. 5B) (Winfree 1975, 1980). In the former mechanism, individual clocks become arrhythmic, i.e., the amplitude of the individual cells is substantially attenuated by the application of the critical light pulse. In contrast, in the latter mechanism of desynchronization, the phases of individual clocks are diversified by the critical light pulse. Although both mechanisms can explain substantial suppression of the multi-cell-level amplitude of circadian rhythm, there is a fundamental difference in dynamical properties between the two in that the oscillations of individual cells are impaired in the former, whereas individual cells maintain their oscillations in the latter. Importantly, although many researchers have observed multi-cell-level singularity behavior in various organisms, it remains elusive whether arrhythmicity or desynchronization of individual clocks underlies the singularity behavior of circadian clocks.

Determination of the underlying mechanism for singularity behaviors of circadian clocks may require adjustable perturbation, because the ability of a critical stimulus to drive circadian clocks into singularity depends on its tim-
A

B

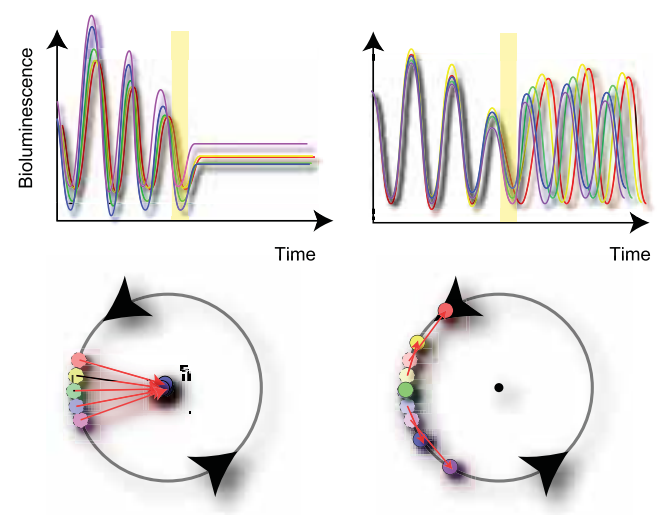

C

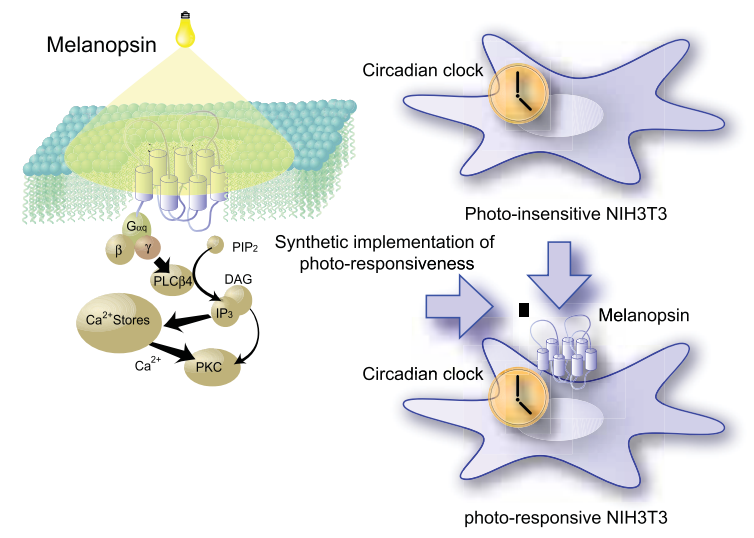

Figure 5. $(A-B)$ Schematic diagrams of two alternative singlecell-level mechanisms for multi-cell-level singularity behavior. Arrhythmicity $(A)$ and desynchronization $(B)$ of individual cellular clocks are indicated. $(C)$ Synthetic implementation of photoresponsiveness within mammalian clock cells. Schematic representation of melanopsin-dependent photoresponsive NIH$3 \mathrm{~T} 3$ cells and the known Gq signaling pathway. (Modified from Ukai et al. 2007.)

ing and strength. Various stimuli, such as reagents and temperature, have been reported to directly reset mammalian cellular clocks (Balsalobre et al. 1998, 2000a,b; Akashi and Nishida 2000; Yagita and Okamura 2000; Yagita et al. 2001; Brown et al. 2002; Hirota et al. 2002; Tsuchiya et al. 2003, 2005). Unfortunately, it is difficult, but not impossible, for these factors to achieve the requisite flexibility in timing and strength. In contrast to perturbations achieved by the use of reagents or temperature change, photoperturbation provides an ideal range of adjustability in timing and strength. Although most mammalian cells cannot sense light, recent studies have shown that mammalian cells (Neuro-2a; HEK293) become photoresponsive following the introduction of an exogenous G-protein-coupled photoreceptor, melanopsin (Melyan et al. 2005; Qiu et al. 2005). It was reported that photostimulation of melanopsin triggers a release of intracellular calcium mediated through the $\mathrm{G}_{\mathrm{q}}$-protein signaling pathway, and, importantly, there are several reports that mammalian cellular clocks can be reset by the $\mathrm{G}_{\mathrm{q}}$-protein signaling pathway involving a release of intracellular calcium (Balsalobre et al. 2000a; Tsuchiya et al. 2005). These 
results suggest that melanopsin-dependent photoperturbation may enable the adjustable and quantitative perturbation of mammalian cellular clocks by changing intracellular calcium level.

To experimentally reveal the underlying mechanism of singularity behavior of mammalian cells, we synthetically implemented photoresponsive mammalian cells by exogenously introducing a $\mathrm{G}_{\mathrm{q}}$-protein-coupled photoreceptor-melanopsin in our recent study (Fig. 5C), see also our recent study (Ukai et al. 2007). We then devised a high-throughput monitoring system with a light-exposure unit to continuously and quantitatively monitor the effect of photoperturbation on the state of cellular clocks. Using this system, we revealed that a critical light pulse drives cellular clocks into a singularity behavior in which robust circadian rhythmicity can be abolished after a certain stimulus. Theoretical analysis and subsequent singlecell-level observation consistently predicted and directly proved that the desynchronization of individual cellular clocks underlies this singularity behavior. We also constructed a theoretical framework to explain why singularity behaviors have been experimentally observed in various organisms and proposed desynchronization as a plausible mechanism for the observable singularity of circadian clocks. Importantly, these in cellulo and in silico findings are further supported by our in vivo observations that desynchronization actually underlies the multi-celllevel amplitude decrease in the rat SCN induced by the critical light pulses that can predispose organisms to transient amplitude decrease in their locomotor activity.

Historically speaking, to elucidate the underlying mechanism of singularity behaviors, Arthur T. Winfree and other investigaters conducted a two-pulse experiment and revealed the "unclocklike" behavior of circadian clock, in which the critical light pulse inducing the singularity behavior seemed to decrease the amplitude of circadian clock without affecting its frequency, apparently arguing against both the simple limit-cycle model and its resulting prediction that the arrhythmicity would underlie the singularity behavior. To explain this "unclocklike" behavior of circadian clock, he proposed the "clockshop" hypothesis, in which an organism-level circadian clock consists of multiple circadian oscillators with substantial fluctuations, and predicted that the desynchronization of individual circadian oscillators would underlie the singularity behavior (multi-cell-level amplitude decrease). He was unable to directly test this prediction, however, as there was no way to observe single-cell-level circadian rhythmicity at that time. In our recent study (Ukai et al. 2007), his prediction on the desynchronization was directly proved at least in the mammalian circadian system more than 30 years after he originally proposed it in his review (Winfree 1975).

\section{Design of Clocks}

The next step is system design-reconstruction and design of new systems based on the design principles that have been revealed through the efforts of a combination strategy of system identification, system analysis, and system control. In this stage, we can validate the sufficiency of the hypothesis derived from the identified structure or observed dynamics. To test the sufficiency of the design principles derived from the identified transcriptional circuits underlying mammalian circadian clocks (Fig. 3B), we are trying to extend our in cellulo cycling assay system (Fig. 3A) to the "physical simulator," with which we will be able to implement artificial transcriptional circuits of interest. We are planning to use this in cellulo system to prove the sufficiency of the components we predict in the natural circadian phase-controlling mechanism.

Alternatively, we can also take a radical and fundamental approach for system design of mammalian circadian clocks. In the next section, we introduce, as a radical approach, our current project on the in vitro reconstruction of the mammalian circadian clock from scratch.

Reconstitution of clocks. The molecular mechanisms underlying circadian clocks in many organisms have been studied for many years (Dunlap 1999; Young and Kay 2001; Reppert and Weaver 2002). Most of the molecularlevel observations in previous studies suggest that every clock system has the translational-transcriptional negative feedback loop as a central oscillator, in which positive regulators such as BMAL1/CLOCK in mammals directly or indirectly activate the transcription of its negative regulators such as Pers/Crys, via a cis-acting DNAelement-like E box (Gekakis et al. 1998; Kume et al. 1999; Shearman et al. 2000).

Although comprehensive studies about the molecular mechanism of clocks have been reported, the basic mechanism for the fundamental nature of circadian clocks is still unclear. Specifically, the mechanism for autonomous oscillation, the most fundamental mechanism of clocks, remains to be fully understood.

Recently, these fundamental questions in eukaryotic clocks have become evident from studies concerning the circadian clock of cyanobacteria, known as the simplest organism possessing a circadian clock. Although ubiquitous molecular behaviors concerning the circadian clock, such as negative feedback regulation of clock genes, circadian oscillation of accumulation of mRNA and clock proteins, and phosphorylation of clock proteins, are also observed in cyanobacteria, the robust circadian oscillation of the phosphorylation state of KaiC, a circadian clock protein, was reconstituted by mixing only three cyanobacterial clock proteins and ATP in a test tube (Fig. 6) (Nakajima et al. 2005). The circadian oscillation of KaiC phosphorylation was therefore proved to be the central oscillator of the cyanobacterial circadian clock. A number of elegant studies about the cyanobacterial circadian clock evoked the importance of using a biochemical approach of the circadian clock for fundamental questions, such as autonomous oscillation and temperaturecompensation.

Clock-related genes and proteins have been almost completely identified in some model organisms such as mammals, Drosophila, and Neurospora, as well as in cyanobacteria (Dunlap 1999; Young and Kay 2001; Reppert and Weaver 2002; Dunlap et al. 2004). In addition to the identification of clock-related components, we have clarified the dynamic nature of circadian clocks. 


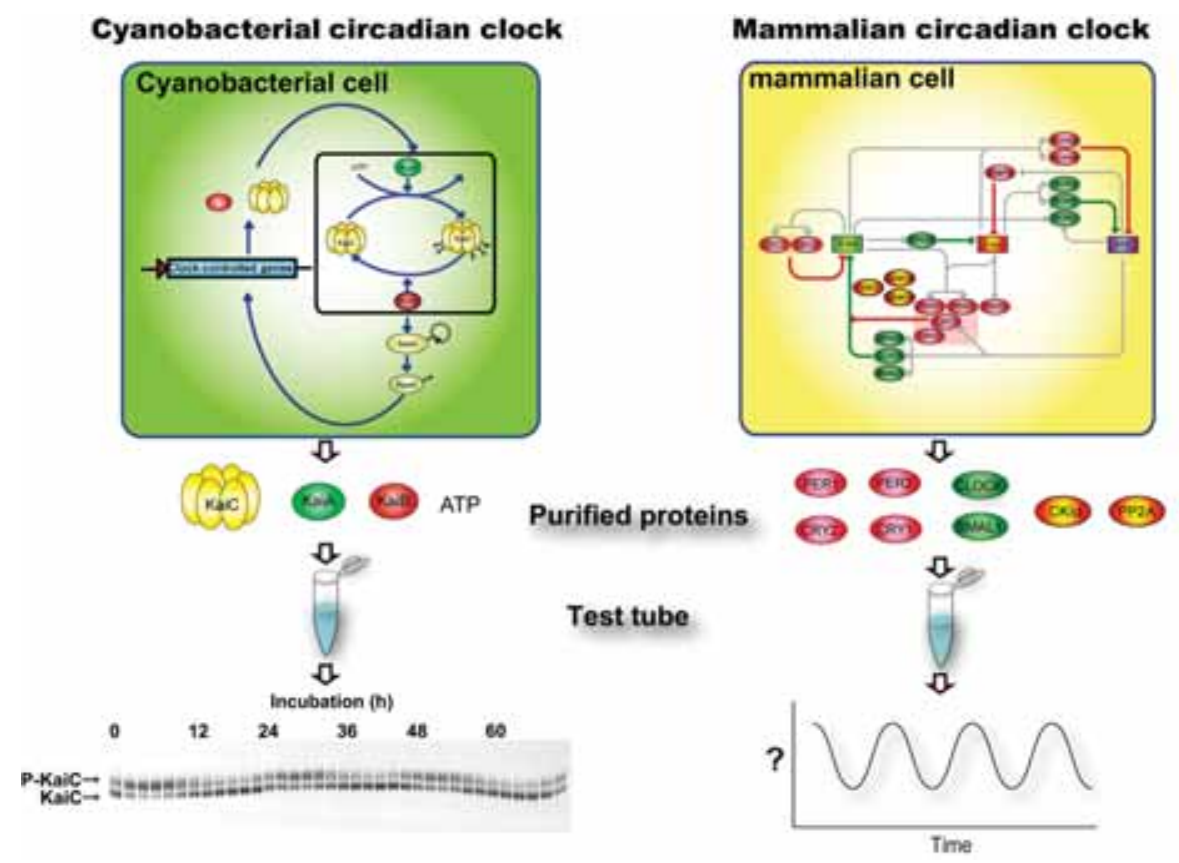

Figure 6. Biochemical approach to the fundamental questions of the circadian clock. KaiC, one of the three cyanobacterial clock proteins (KaiA, KaiB, and $\mathrm{KaiC}$ ), has both autokinase and autophosphatase activity, and its phosphorylation state shows circadian rhythm in vivo. When three Kai proteins and ATP are mixed in a test tube, temperature-compensated KaiC phosphorylation rhythm is reconstituted (left). Because the biochemical properties of the mammalian clock proteins are limited, it is thought that the fundamental nature of the mammalian circadian clock is also dependent on these clock proteins (right). (Modified from Nakajima et al. 2005; panel on the periodic KaiC phosphorylation is courtesy of Dr. M. Nakajima.)

These circumstances in circadian clock studies led us to reevaluate the negative feedback model of the circadian clock by biochemical methods. We have succeeded in the purification of some clock proteins through the expression system of Escherichia coli. Furthermore, we are now trying to characterize some basic reactions in the mammalian circadian clock, such as the enzymatic properties of casein kinase I $\varepsilon$ (CKIE) (Lowrey et al. 2000) and some protein-protein interactions. Although the mammalian circadian clock system is more complicated than that of cyanobacteria, we are now trying to reconstitute the mammalian circadian clock to demonstrate the essential mechanism of autonomous circadian oscillation and the other fundamental questions.

\section{PERSPECTIVES: SYSTEM-LEVEL QUESTIONS}

In the previous sections, we introduced a series of systems-biological approaches as well as their application to the specific questions such as singularity behaviors of mammalian circadian clocks. This has provided us with the groundwork to take a further step forward. It is high time to fully integrate these approaches to realize a system-level understanding of the mammalian circadian clock. The success of these approaches will therefore be measured by the importance and number of system-level questions that are solved. In the following sections, we list several system-level questions in mammalian circadian clocks (Fig. 7). We first describe the dynamical problems related to the core of clocks, including the delay in feedback repression; high-amplitude oscillations generated by transcriptional response of nonlinearity; temperature-compensation in elementary processes of the circadian oscillation; and synchronization of clocks against inevitable fluctuations of phase and period in multiple circadian oscillators. We then introduce information problems related to clocks in the environment, including the internal representation of light change, especially through a mechanism known as perfect adaptation and the internal representation of day length by photoperiodism.

\section{Core of Clocks}

In the mammalian circadian clocks, it is assumed that the delay in transcriptional feedback repression has a pivotal role in generating circadian oscillations based on the basic control theory. However, the underlying molecular mechanism to generate the delay still remains elusive. We first describe this dynamical question on delay. Nonlinearity in transcriptional feedback repression also has an important role in generating high-amplitude circadian oscillation itself as well as the high-amplitude output oscillations. Thus, as the second dynamical question, we pick up this nonlinearity problem. In addition to these dynamical properties, the core of mammalian circadian clocks also exhibit well-defined dynamical properties, including temperaturecompensation and synchronization of multiple cellular clocks. We describe these two dynamical questions in the subsequent sections. 


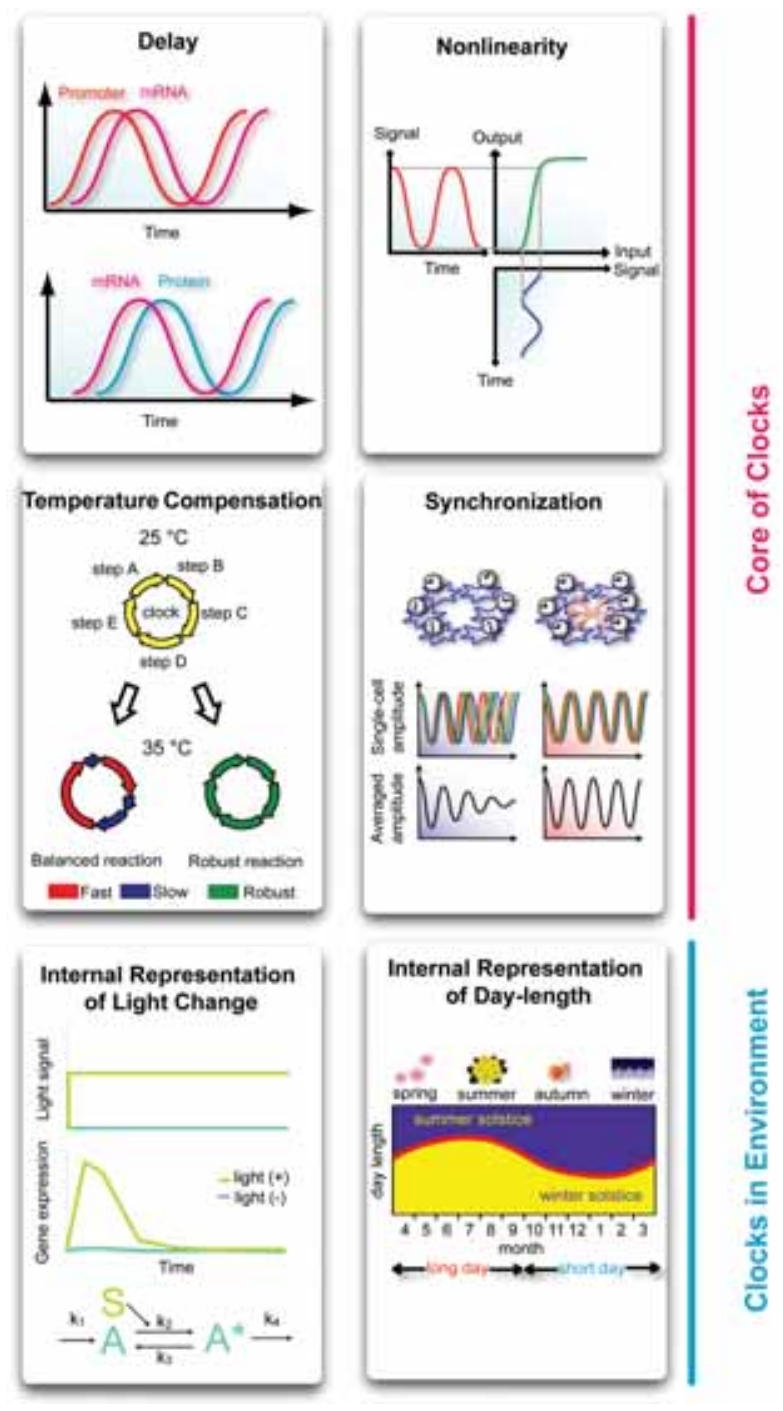

Figure 7. System-level questions in mammalian circadian clocks. Questions related to core of clocks: Delay in feedback repression seems to have an important role in the generation of oscillations. Although the nonlinearity involved in generating a high amplitude of transcription has been implicitly supposed in mammalian circadian clock, its mechanism so far remains unsolved. Although temperature-compensation is one of the most mysterious dynamical properties of clocks, its mechanism so far remains unsolved. The synchronization mechanism to generate synchrony of the multiple cellular clocks has been proposed theoretically but is not yet proven. Questions related to clocks in environment: Central clock tissue can internally represent a change of environmental light signal probably through perfect adaptation. Several mechanisms for perfect adaptation have been proposed theoretically but are not yet proven in mammalian circadian clocks. Organisms can also internally represent day length through photoperiodism. Although a gating mechanism is expected, its mechanism so far remains unsolved.

Delay. System identification and system analysis of mammalian circadian clocks revealed that transcriptional feedback repression mediated through the E box has an important role in generating transcriptional circadian oscillation. Interestingly, this transcriptional feedback repression accompanies delay in elementary processes, which is consistent with the basic control theory predicting that the negative feedback loop with a certain delay can generate the oscillation with twofold period of delay. In mammalian circadian clocks, delay has been observed at least in two processes (Fig. 7, top right panel). One is the delay between mRNA amounts and protein amounts. For example, expression levels of Perl and Per2 mRNA are several hours earlier than those of PER1 and PER2 proteins (Lee et al. 2001), respectively. According to the basic control theory, this delay between mRNA and protein may be one of the determinants of duration of circadian oscillation, although there has so far been no definitive proof. The other process is the delay between promoter activity and mRNA amount. For example, promoter activity of Cryl is several hours earlier than the expression level of Cryl mRNA (Etchegaray et al. 2003; Ueda et al. 2005). Because the Cryl gene is one of the strongest repressors for E-box-mediated transcription, this delay also seems to be one of the determinants of duration of circadian oscillation.

A delayed process, where an input signal is just shifted to an output signal by a certain period without change of 
its shape, is completely different from a merely slow process, where an input signal is usually transformed to a dulled output signal. Thus, the generation of delay requires certain molecular mechanisms. However, molecular mechanisms to generate delays and hence oscillations still remain elusive in mammalian circadian clocks.

Nonlinearity. Nonlinearity is another dynamical property closely associated with delay because a slow process with certain nonlinearity can restore the original sharp signal from a dulled signal through amplification (Fig. 7, top right panel). Sigmoidal response (Fig. 7, top right panel), one of the nonlinear responses, is important for this amplification. Sigmoidal response is also important to generate high-amplitude circadian oscillations in clock output where a sharp input signal is usually dulled through a transfer process. Such sigmoidal responses can amplify the dulled signal to restore the original sharp signal and hence generate high-amplitude outputs.

Several molecular mechanisms can be expected to achieve a sigmoidal response. One class of molecular mechanisms is called as cooperativity (Monod et al. 1963), where multiple subunits work together to accomplish the entire process. In this cooperativity process, a Hill coefficient, which describes the strength of nonlinearity in a sigmoidal response, is usually smaller than the number of subunits, and hence usually approximately 2 (Hill coefficient 1 indicates a linear response). On the other hand, the other class of molecular mechanisms called ultrasensitivity (Goldbeter and Koshland 1981) has a potential to generate strong nonlinearity, with a Hill coefficient of more than 2 . This switch-like behavior, where the output signal increases abruptly when an input signal exceeds a certain threshold, was originally proposed theoretically in antagonistic enzymatic reactions, where an enzyme competes with another enzyme acting on the same molecule (Goldbeter and Koshland 1981). However, molecular mechanisms to generate such a sigmoidal response and hence high-amplitude oscillations still remain elusive in mammalian circadian clocks.

Temperature-compensation. A robust 24-hour period against environmental changes, such as temperature and nutrition, is an important element for the circadian clock. The robustness of the period against temperature change is known as temperature-compensation, which is one of the intriguing aspects of the circadian clock. Typical biochemical reactions, such as enzymatic reactions, show temperature dependence, which is represented by a Q10 value of about 2 . In contrast, the period of circadian rhythm is independent of or compensated against temperature change (Q10 value of the period is about 1.). The importance of temperature-compensation in poikilotherm can be easily understood. Although this aspect of nature in the mammalian circadian clock has been controversial, it has been confirmed in cultured mammalian cells (Izumo et al. 2003; Tsuchiya et al. 2003). Temperaturecompensation has proved to be one of the general elements of circadian clocks from cyanobacteria to humans. Despite our increasing knowledge of the molecular mechanism of circadian clocks, however, it is difficult to explain how circadian clocks sustain such a constant period against temperature change.

Theoretical studies (Ruoff et al. 1997; Kurosawa and Iwasa 2005) have proposed a balanced reaction model (Fig. 7, middle left panel) for explaining this nature, in which increasing kinetic parameters of some reactions in the negative feedback loop lead to a shortening of the period and others lead to prolonging the period. As a result, the period of the circadian rhythm is unchanged against temperature change by a balance between positive and negative effects of reactions on the period. These theoretical explanations seem to be plausible, but the balance of effects on the period length between basic reactions can be easily broken by perturbations, such as inhibitors and point mutations on clock proteins. Furthermore, these theoretical models seem inconsistent with the fact that many circadian clock mutants show a diverse period but sustain temperature-compensation. How do circadian clocks acquire the robustness against temperature change?

There are some implications for understanding the mechanism in studies of the cyanobacterial circadian clock. The circadian rhythm of KaiC phosphorylation can be reconstituted in a test tube, demonstrating that the KaiC phosphorylation rhythm is the central oscillator of the cyanobacterial circadian clock (Nakajima et al. 2005). The reaction rate of KaiC phosphorylation in a test tube is unaffected by temperature change despite the fact that it is a biochemical reaction. These observations indicate that the robustness of circadian oscillation in cyanobacterial cells depends on the biochemical properties of three clock proteins. Although the mechanism of the KaiC phosphorylation cycle remains unclear, these findings suggest a robust reaction model (Fig. 7, middle left panel) for temperature-compensation of circadian clocks, at least in cyanobacteria.

In summary, there exist two possible models of temperature-compensations of the circadian clock. In the balanced reaction model, increasing kinetic parameters of some basic reactions lead to shortening the period, and those of other basic reactions lead to prolonging the period. These effects are cancelled out so that the period is sustained constantly. In the balanced reaction model, temperature-compensation is expected as a emergent network property of multiple clock components. In the robust reaction model, such as the cyanobacterial circadian clock, temperature-compensation of the circadian clock is caused by reactions, of which kinetic parameters are independent of temperature change. In the robust reaction model, temperature-compensation is expected as a molecular property of clock components. According to this model, the system-level understanding of temperature-compensation extensively depends on the investigation of biochemical property of clock components.

Synchronization of clocks. The core of mammalian circadian clocks located in the SCN consists of multiple autonomous single-cell oscillators. The individual cellular oscillators in the SCN produce coherent circadian rhythm despite the inevitable internal noise. However, the synchronization mechanism to couple individual cellular oscillators is largely unknown. As a possible model of the 
intercellular synchronization of circadian cellular clocks, we previously presented a multicellular stochastic model with an intercellular synchronization factor that emulates the synchronization of individual cellular oscillators (Ueda et al. 2002a). In this model, owing to a synchronizing factor, the neighboring cells correct each other's differences to stay in the proper rhythm, and thus, coherent oscillation of individual cellular clock is retained even in the presence of internal noise (Fig. 7, middle right panel). On the other hand, in the model without such a synchronizing factor, coherent oscillation of individual cellular clocks is abolished despite the identical initial conditions. The averaged amplitude is reduced due to the internal noise and the absence of external time cues such as light and temperature (Fig. 7, middle right panel).

In our model, a synchronization factor is supposed to be secreted from the individual cellular oscillators and then affect the circadian state of neighboring cells. The model predicts that if the synchronization factor is secreted during the subjective day, then the synchronization factor will induce the light-type phase-response in the neighboring cells (Ueda et al. 2002a). On the other hand, it is predicted that if the synchronization factor is secreted during the subjective night, then it will induce the dark-type phase-response in the neighboring cells.

Interestingly, recent studies reported a candidate synchronization factor, vasoactive intestinal polypeptide (VIP), which seems to possess the predicted dynamical properties described above. VIP is a peptide-type neurotransmitter that is secreted during the subjective day in the SCN and is known to induce a light-type phase-response in the circadian clock in a cultured SCN slice (Reed et al. 2001). Moreover, the VIP receptor VPAC2 is also expressed in the SCN. Actually, it has been reported that loss of VIP or VPAC2 disrupted synchrony between each oscillator neuron in the SCN (Aton et al. 2005; Maywood et al. 2006). Seen in this light, VIP has an important role in provoking the synchronous oscillation of cells at the SCN via VPAC2 receptor signaling. If our theory is correct, we may be able to reconstruct the synchronization of individual cellular oscillators in cultured cells such as Rat-1 and NIH-3T3, which exhibits no detectable synchronization among cells, by exogenously expressing VIP and the VPAC2 receptor under the control of a clock-controlled promoter at the appropriate timing in cultured cells. The theory predicts that VIP expressed during the subjective day will induce the efficient coupling among individual cellular oscillators, but VIP expressed at other times such as subjective night will not. The experimental verification of these predictions may lead to design principles in the synchronization of mammalian circadian clocks.

\section{Clocks in Environment}

Mammalian circadian clocks are surrounded by an environment and thereby receive external stimuli such as light and temperature to extract environmental information on the state of the earth. In the following sections, we discuss how the living organism accurately extracts and internally represents change of light signals and the day length.
Internal representation of light change: Perfect adaptation. Living organisms sense changes of external environment and entrain their internal circadian clocks by utilizing environmental change as a time cue. The most well-known time cue is light, and dawn and dusk light mark the advance and delay of the circadian clock, respectively. These rapid illumination changes that occur at dawn and dusk act on the resetting of circadian rhythm in the SCN, which is known as nonparametric entrainment (Johnson et al. 2003). How does the central clock tissue internally represent the change of the light signals in order to achieve nonparametric entrainment?

One of the biologically plausible mechanisms to sense environmental change is known as perfect adaptation (Hao et al. 2007), where the system can sense not the absolute value but the relative change of external signals. Three possible mathematical models have been proposed for (near) perfect adaptation: (1) feed-forward loop, (2) feedback loop, and (3) activation-dependent inactivation. The feedforward loop model has activation and inactivation pathways to receptor A from signal S. Signal S both activates and inactivates receptor A. Suppose that there are fast activation and slow inactivation of receptor $\mathrm{A}$, then the active form of receptor $A^{*}$ would be perfectly adapted, i.e., $A^{*}$ would rapidly respond to the step-up of signal S but quickly go back to its original level. It is also noteworthy that both activation and inactivation of receptor $\mathrm{A}$ by signal S must have a similar dependency on signal $\mathrm{S}$ in order to achieve the perfect adaptation in the feed-forward loop model. The second model is the feedback loop model where signal $\mathrm{S}$ activates receptors, and then the active form receptor $\mathrm{A}^{*}$ inhibits the receptor activation or synthesis. We note that the feedback loop model only achieves near perfect adaptation, where $A *$ rapidly responds to the step-up of signal $\mathrm{S}$ and goes back near to its original level (but this is not perfect). The third and most robust model is the active-dependent inactivation model, where signal $\mathrm{S}$ activates the receptor. Then, only activated receptor $\mathrm{A}^{*}$ is degraded or inactivated (Fig. 7, bottom left panel).

To solve this information problem on the internal representation of light change, it will be a limiting process to identify the "perfect adaptation" gene, which only responds to the change of the light. When the step function of light is applied, this type of genes will be rapidly induced once but quickly go back to its original expression level (Fig. 7, bottom left panel). Following the identification of such genes, precise measurement and quantitative perturbation of its expression dynamics will be critical for the system-level understanding of its design principle.

Internal representation of day length: Photoperiodism. Living organisms also measure the day length (i.e., the duration of daily light time) and hence sense the season of external environment because day length is longer in summer and shorter in winter. This alternation of day length induces a seasonal physiological and metabolic change called photoperiodism (Dunlap et al. 2004). Living organisms are believed to demonstrate photoperiodism through circadian clock function because only certain periods in a day (photoinducible phase) are sensitive to light signals and have a critical role in mea- 
surement of day length. However, it is still largely unknown how the specific brain region can internally represent the day length through interplay between the circadian clock and light (or dark) signals, especially in mammals (Fig. 7, bottom right panel).

In photoperiodism research on animals, birds such as the Japanese quail are often used because of their dynamic and rapid response to day length change. In birds, it is known that light exposure in a certain phase (photoinducible phase) can induce a photoperiodic response. Japanese quail have an approximately 4-hour photoinducible phase that starts about 12-16 hours after the beginning of the light period. When the day length becomes longer (season changes to breeding period), female Japanese quail begin to lay eggs and males' testicles start to mature. During the breeding period, their testis enlarge to more than 100 times that of the nonbreeding period. At the hormonal regulation level, triiodothyronine (T3), an active form of enzyme converted from deiodination of thyroxine (T4) prohormone via Dio2 activity, promotes secretion of gonadotropin-releasing hormone $(\mathrm{GnRH})$ and hence testicular maturation.

Scientific efforts to reveal the induction mechanism of photoperiodic reaction in Japanese quail have achieved great success in recent years. To summarize, the median eminence (ME) in the hypothalamus was found to be the responsible area for photoperiodism formation. In the ME, light stimulation induces Dio2 at the photoinducible phase. On the other hand, Dio2 is not induced in the nonphotoinducible phase. As clock gene oscillation has been reported in these areas, the circadian clock is also thought to exist in these regions. As there is also a time-dependent gating system (i.e., photoinducible phase), the relationship between Dio2 gene regulation and the circadian clock system has already been the subject of discussion (Yoshimura et al. 2003). In mammals, the pineal body, which is known as a melatonin-synthesizing organ, is important in photoperiodic reaction. Because melatonin synthesis occurs during the night and is repressed by light, melatonin level represents the dark length information. Interestingly, expression of Dio2 in ME, which highly expresses a melatonin receptor, is also under the influence of melatonin in mammals. Thus, the mammals and birds share a photoperiodic mechanism.

To solve this information problem on the internal representation of day length and season, it will be a limiting process to identify the "photoperiodic" gene, which is rapidly induced (or repressed) in response to the longer day length, and to regulate Dio2 and other downstream genes. After the identification of such a gene, precise measurement and quantitative perturbation of its gating expression dynamics will be critical for the system-level understanding of its design principle.

\section{CONCLUSION}

Following the identification of the key clock genes, there was increasing demand for higher-order understanding of design principles in mammalian circadian clocks. In this chapter, we described several approaches, beginning with comprehensive identification (system identification) and quantitative analysis (system analysis) of individual clock components and their networked interactions, leading to the ability to control existing systems toward the desired state (system control) and the design of new ones based on an understanding of structure and underlying dynamical principles (system design). We also listed several dynamical and information problems including delay, nonlinearity, temperature-compensation, and synchronization of mammalian circadian oscillator(s), as well as the internal representation of light change through perfect adaptation and internal representation of day length through photoperiodism in mammals. We strongly believe that it is now high time to fully integrate the systems-biological approaches for the solution of the system-level questions.

\section{ACKNOWLEDGMENTS}

We thank our collaborators. System identification of clocks: collaboration with Drs. W. Chen, A. Adachi, H. Wakamatsu, S. Hayashi, T. Takasugi, M. Nagano, K. Nakahama, Y. Suzuki, S. Sugano, M. Iino, Y. Shigeyoshi, S. Hashimoto, M. Sano, and M. Machida. System analysis of clocks: collaboration with Drs. T.K. Sato, R.G. Yamada, H. Ukai, J.E. Baggs, L.J. Miraglia, T.J. Kobayashi, D.K. Welsh, S.A. Kay, and J.B. Hogenesch. System control of clocks: collaboration with Drs. H. Ukai, T.J. Kobayashi, M. Nagano, K. Masumoto, M. Sujino, T. Kondo, K. Yagita, and Y. Shigeyoshi. System design of clocks: collaboration with Drs. M. Nakajima, M. Ishida, M. Ukai-Tadenuma, and T. Kasukawa. Molecular-timetable method: collaborative work with Drs. W. Chen, Y. Minami, S. Honma, K. Honma, M. Iino, and S. Hashimoto. Theoretical study on synchronization of clocks: collaboration with K. Hirose and M. Iino. We also thank Drs. I. Nikaido, H. Ukai, R.G. Yamada, T.J. Kobayashi, M. Nakajima, and Y. Minami as well as A. Wada for creation of the figures and critical reading of manuscript. This research was supported by intramural grant-in-aid from the Center for Developmental Biology (CDB), Director's Fund from CDB, President's Fund from Riken, grant-in-aid for Genome-network Project and Scientific Research on Priority Areas "Systems Genomics" from MEXT, Japan, and grant-in-aid for NEDO project from METI, Japan.

\section{REFERENCES}

Akashi M. and Nishida E. 2000. Involvement of the MAP kinase cascade in resetting of the mammalian circadian clock. Genes Dev. 14: 645.

Aton S.J., Colwell C.S., Harmar A.J., Waschek J., and Herzog E.D. 2005. Vasoactive intestinal polypeptide mediates circadian rhythmicity and synchrony in mammalian clock neurons. Nat. Neurosci. 8: 476.

Bae K., Jin X., Maywood E.S., Hastings M.H., Reppert S.M., and Weaver D.R. 2001. Differential functions of mPer1, mPer2, and mPer3 in the SCN circadian clock. Neuron 30: 525.

Balsalobre A., Damiola F., and Schibler U. 1998. A serum shock induces circadian gene expression in mammalian tissue culture cells. Cell 93: 929.

Balsalobre A., Marcacci L., and Schibler U. 2000a. Multiple signaling pathways elicit circadian gene expression in cultured Rat-1 fibroblasts. Curr. Biol. 10: 1291.

Balsalobre A., Brown S.A., Marcacci L., Tronche F., Kellendonk C., Reichardt H.M., Schutz G., and Schibler U. 
2000b. Resetting of circadian time in peripheral tissues by glucocorticoid signaling. Science 289: 2344.

Brown S.A., Zumbrunn G., Fleury-Olela F., Preitner N., and Schibler U. 2002. Rhythms of mammalian body temperature can sustain peripheral circadian clocks. Curr. Biol. 12: 1574.

Bunger M.K., Wilsbacher L.D., Moran S.M., Clendenin C., Radcliffe L.A., Hogenesch J.B., Simon M.C., Takahashi J.S., and Bradfield C.A. 2000. Mop3 is an essential component of the master circadian pacemaker in mammals. Cell 103: 1009.

Covington M.F., Panda S., Liu X.L., Strayer C.A., Wagner D.R., and Kay S.A. 2001. ELF3 modulates resetting of the circadian clock in Arabidopsis. Plant Cell 13: 1305.

Dunlap J.C. 1999. Molecular bases for circadian clocks. Cell 96: 271.

Dunlap J.C., Loros J.J., and DeCoursey P.J., eds. 2004 Chronobiology: Biological timekeeping. Sinauer Associates, Sunderland, Massachusetts.

Engelmann W., Johnsson A., Karlsson H.G., Kobler R., and Schimmel M.-L. 1978. Attenuation of the petal movement rhythm in kalanchoe with light pulses. Physiol. Plant. 43: 68 .

Etchegaray J.P., Lee C., Wade P.A., and Reppert S.M. 2003. Rhythmic histone acetylation underlies transcription in the mammalian circadian clock. Nature 421: 177.

Falvey E., Marcacci L., and Schibler U. 1996. DNA-binding specificity of PAR and C/EBP leucine zipper proteins: A single amino acid substitution in the C/EBP DNA-binding domain confers PAR-like specificity to C/EBP. Biol. Chem. 377: 797.

Gekakis N., Staknis D., Nguyen H.B., Davis F.C., Wilsbacher L.D., King D.P., Takahashi J.S., and Weitz C.J. 1998. Role of the CLOCK protein in the mammalian circadian mechanism. Science 280: 1564.

Goldbeter A. and Koshland D.E., Jr. 1981. An amplified sensitivity arising from covalent modification in biological systems. Proc. Natl. Acad. Sci. 78: 6840.

Hao N., Behar M., Elston T.C., and Dohlman H.G. 2007. Systems biology analysis of G protein and MAP kinase signaling in yeast. Oncogene 26: 3254 .

Harding H.P. and Lazar M.A. 1993. The orphan receptor RevErbA alpha activates transcription via a novel response element. Mol. Cell. Biol. 13: 3113.

Hirota T., Okano T., Kokame K., Shirotani-Ikejima H., Miyata T., and Fukada Y. 2002. Glucose down-regulates Per1 and Per2 mRNA levels and induces circadian gene expression in cultured Rat-1 fibroblasts. J. Biol. Chem. 277: 44244.

Hogenesch J.B., Gu Y.Z., Jain S., and Bradfield C.A. 1998. The basic-helix-loop-helix-PAS orphan MOP3 forms transcriptionally active complexes with circadian and hypoxia factors. Proc. Natl. Acad. Sci. 95: 5474.

Honma S. and Honma K. 1999. Light-induced uncoupling of multioscillatory circadian system in a diurnal rodent, Asian chipmunk. Am. J. Physiol. 276: R1390.

Honma S., Kawamoto T., Takagi Y., Fujimoto K., Sato F., Noshiro M., Kato Y., and Honma K. 2002. Dec1 and Dec2 are regulators of the mammalian molecular clock. Nature 419: 841 .

Huang G., Wang L., and Liu Y. 2006. Molecular mechanism of suppression of circadian rhythms by a critical stimulus. EMBO J. 25: 5349 .

Izumo M., Johnson C.H., and Yamazaki S. 2003. Circadian gene expression in mammalian fibroblasts revealed by real-time luminescence reporting: Temperature compensation and damping. Proc. Natl. Acad. Sci. 100: 16089.

Jewett M.E., Kronauer R.E., and Czeisler C.A. 1991. Lightinduced suppression of endogenous circadian amplitude in humans. Nature 350: 59.

Johnson C.H. and Kondo T. 1992. Light pulses induce "singular" behavior and shorten the period of the circadian phototaxis rhythm in the CW15 strain of Chlamydomonas. J. Biol. Rhythms 7: 313

Johnson C.H., Elliott J.A., and Foster R. 2003. Entrainment of circadian programs. Chronobiol. Int. 20: 741.

King D.P., Zhao Y., Sangoram A.M., Wilsbacher L.D., Tanaka M., Antoch M.P., Steeves T.D., Vitaterna M.H., Kornhauser
J.M., Lowrey P.L., Turek F.W., and Takahashi J.S. 1997. Positional cloning of the mouse circadian clock gene. Cell 89: 641.

Kitano, H. 2002a. Computational systems biology. Nature 420: 206.

. 2002b. Systems biology: A brief overview. Science 295: 1662.

Kume K., Zylka M.J., Sriram S., Shearman L.P., Weaver D.R., Jin X., Maywood E.S., Hastings M.H., and Reppert S.M. 1999. mCRY1 and mCRY2 are essential components of the negative limb of the circadian clock feedback loop. Cell 98: 193.

Kurosawa G. and Iwasa Y. 2005. Temperature compensation in circadian clock models. J. Theor. Biol. 233: 453.

Lee C., Etchegaray J.P., Cagampang F.R., Loudon A.S., and Reppert S.M. 2001. Posttranslational mechanisms regulate the mammalian circadian clock. Cell 107: 855.

Lipshutz R.J., Fodor S.P., Gingeras T.R., and Lockhart D.J. 1999. High density synthetic oligonucleotide arrays. Nat. Genet. (suppl. 1) 21: 20.

Lowrey P.L., Shimomura K., Antoch M.P., Yamazaki S., Zemenides P.D., Ralph M.R., Menaker M., and Takahashi J.S. 2000. Positional syntenic cloning and functional characterization of the mammalian circadian mutation tau. Science 288: 483.

Malinowski J.R., Laval-Martin D.L., and Edmunds L.N., Jr. 1985. Circadian oscillators, cell cycles, and singularities: Light perturbations of the free-running rhythm of cell division in Euglena. J. Comp. Physiol. B 155: 257.

Maywood E.S., Reddy A.B., Wong G.K., O'Neill J.S., O'Brien J.A., McMahon D.G., Harmar A.J., Okamura H., and Hastings M.H. 2006. Synchronization and maintenance of timekeeping in suprachiasmatic circadian clock cells by neuropeptidergic signaling. Curr. Biol. 16: 599.

Melyan Z., Tarttelin E.E., Bellingham J., Lucas R.J., and Hankins M.W. 2005. Addition of human melanopsin renders mammalian cells photoresponsive. Nature 433: 741.

Mitsui S., Yamaguchi S., Matsuo T., Ishida Y., and Okamura H. 2001. Antagonistic role of E4BP4 and PAR proteins in the circadian oscillatory mechanism. Genes Dev. 15: 995.

Monod J., Changeux J.P., and Jacob F. 1963. Allosteric proteins and cellular control systems. J. Mol. Biol. 6: 306.

Nakajima M., Imai K., Ito H., Nishiwaki T., Murayama Y., Iwasaki H., Oyama T., and Kondo T. 2005. Reconstitution of circadian oscillation of cyanobacterial KaiC phosphorylation in vitro. Science 308: 414.

Okamura H., Miyake S., Sumi Y., Yamaguchi S., Yasui A., Muijtjens M., Hoeijmakers J.H., and van der Horst G.T. 1999. Photic induction of mPer1 and mPer2 in cry-deficient mice lacking a biological clock. Science 286: 2531.

Panda S., Hogenesch J.B., and Kay S.A. 2002. Circadian rhythms from flies to human. Nature 417: 329.

Preitner N., Damiola F., Lopez-Molina L., Zakany J., Duboule D., Albrecht U., and Schibler U. 2002. The orphan nuclear receptor REV-ERBalpha controls circadian transcription within the positive limb of the mammalian circadian oscillator. Cell 110: 251.

Qiu X., Kumbalasiri T., Carlson S.M., Wong K.Y., Krishna V., Provencio I., and Berson D.M. 2005. Induction of photosensitivity by heterologous expression of melanopsin. Nature 433: 745.

Reed H.E., Meyer-Spasche A., Cutler D.J., Coen C.W., and Piggins H.D. 2001. Vasoactive intestinal polypeptide (VIP) phase-shifts the rat suprachiasmatic nucleus clock in vitro. Eur. J. Neurosci. 13: 839.

Reick M., Garcia J.A., Dudley C., and McKnight S.L. 2001. NPAS2: An analog of clock operative in the mammalian forebrain. Science 293: 506 .

Reppert S.M. and Weaver D.R. 2002. Coordination of circadian timing in mammals. Nature 418: 935.

Ruoff P., Rensing L., Kommedal R., and Mohsenzadeh S. 1997. Modeling temperature compensation in chemical and biological oscillators. Chronobiol. Int. 14: 499.

Sato T.K., Panda S., Miraglia L.J., Reyes T.M., Rudic R.D., 
McNamara P., Naik K.A., FitzGerald G.A., Kay S.A., and Hogenesch J.B. 2004. A functional genomics strategy reveals Rora as a component of the mammalian circadian clock. Neuron 43: 527.

Sato T.K., Yamada R.G., Ukai H., Baggs J.E., Miraglia L.J., Kobayashi T.J., Welsh D.K., Kay S.A., Ueda H.R., and Hogenesch J.B. 2006. Feedback repression is required for mammalian circadian clock function. Nat. Genet. 38: 312.

Shearman L.P., Sriram S., Weaver D.R., Maywood E.S., Chaves I., Zheng B., Kume K., Lee C.C., van der Horst G.T., Hastings M.H., and Reppert S.M. 2000. Interacting molecular loops in the mammalian circadian clock. Science 288: 1013.

Suzuki Y., Taira H., Tsunoda T., Mizushima-Sugano J., Sese J., Hata H., Ota T., Isogai T., Tanaka T., Morishita S., Okubo K., Sakaki Y., Nakamura Y., Suyama A., and Sugano S. 2001. Diverse transcriptional initiation revealed by fine, large-scale mapping of mRNA start sites. EMBO Rep. 2: 388.

Taylor W., Krasnow R., Dunlap J.C., Broda H., Hastings J.W. 1982. Critical pulses of anisomycin drive the circadian oscillator in Gonyaulax towards its singularity. J. Comp. Physiol. 148: 11 .

Tei H., Okamura H., Shigeyoshi Y., Fukuhara C., Ozawa R., Hirose M., and Sakaki Y. 1997. Circadian oscillation of a mammalian homologue of the Drosophila period gene. Nature 389: 512.

Tomita J., Nakajima M., Kondo T., and Iwasaki H. 2005. No transcription-translation feedback in circadian rhythm of KaiC phosphorylation. Science 307: 251.

Tsuchiya Y., Akashi M., and Nishida E. 2003. Temperature compensation and temperature resetting of circadian rhythms in mammalian cultured fibroblasts. Genes Cells 8: 713.

Tsuchiya Y., Minami I., Kadotani H., and Nishida E. 2005. Resetting of peripheral circadian clock by prostaglandin E2. EMBO Rep. 6: 256.

Ueda H.R., Hirose K., and Iino M. 2002a. Intercellular coupling mechanism for synchronized and noise-resistant circadian oscillators. J. Theor. Biol. 216: 501.

Ueda H.R., Matsumoto A., Kawamura M., Iino M., Tanimura T., and Hashimoto S. 2002b. Genome-wide transcriptional orchestration of circadian rhythms in Drosophila. J. Biol. Chem. 19: 14048.

Ueda H.R., Chen W., Minami Y., Honma S., Honma K., Iino M. and Hashimoto S. 2004. Molecular-timetable methods for detection of body time and rhythm disorders from singletime-point genome-wide expression profiles. Proc. Natl. Acad. Sci. 101: 11227.

Ueda H.R., Hayashi S., Chen W., Sano M., Machida M. Shigeyoshi Y., Iino M., and Hashimoto S. 2005. System-level identification of transcriptional circuits underlying mammalian circadian clocks. Nat. Genet. 37: 187.

Ueda H.R., Chen W., Adachi A., Wakamatsu H., Hayashi S. Takasugi T., Nagano M., Nakahama K., Suzuki Y., Sugano S., Iino M., Shigeyoshi Y., and Hashimoto S. 2002c. A transcription factor response element for gene expression during circadian night. Nature 418: 534

Ukai H., Kobayashi T.J., Nagano M., Masumoto K., Sujino M., Kondo T., Yagita Y., Shigeyoshi Y., and Ueda H.R. 2007. Melanopsin-dependent photo-perturbation reveals desyn- chronization underlying the singularity of mammalian circadian clocks. Nat. Cell Biol. (in press). [AU: Update?]

van der Horst G.T., Muijtjens M., Kobayashi K., Takano R., Kanno S., Takao M., de Wit J., Verkerk A., Eker A.P., van Leenen D., Buijs R., Bootsma D., Hoeijmakers J.H., and Yasui A. 1999. Mammalian Cry1 and Cry2 are essential for maintenance of circadian rhythms. Nature 398: 627.

Vitaterna M.H., Selby C.P., Todo T., Niwa H., Thompson C., Fruechte E.M., Hitomi K., Thresher R.J., Ishikawa T., Miyazaki J., Takahashi J.S., and Sancar A. 1999. Differential regulation of mammalian period genes and circadian rhythmicity by cryptochromes 1 and 2. Proc. Natl. Acad. Sci. 96: 12114

Welsh D.K., Yoo S.H., Liu A.C., Takahashi J.S., and Kay S.A. 2004. Bioluminescence imaging of individual fibroblasts reveals persistent, independently phased circadian rhythms of clock gene expression. Curr. Biol. 14: 2289.

Winfree A.T. 1970. Integrated view of resetting a circadian clock. J. Theor. Biol. 28: 327.

. 1975. Unclocklike behaviour of biological clocks. Nature 253: 315.

. 1980. The geometry of biological time. SpringerVerlag, New York.

Wuarin J. and Schibler U. 1990. Expression of the liver-enriched transcriptional activator protein DBP follows a stringent circadian rhythm. Cell 63: 1257.

Yagita K. and Okamura H. 2000. Forskolin induces circadian gene expression of rPer1, rPer2 and dbp in mammalian rat-1 fibroblasts. FEBS Lett. 465: 79.

Yagita K., Tamanini F., van der Horst G.T., and Okamura H. 2001. Molecular mechanisms of the biological clock in cultured fibroblasts. Science 292: 278.

Yamazaki S., Numano R., Abe M., Hida A., Takahashi R., Ueda M., Block G.D., Sakaki Y., Menaker M., and Tei H. 2000. Resetting central and peripheral circadian oscillators in transgenic rats. Science 288: 682.

Yoo S.H., Ko C.H., Lowrey P.L., Buhr E.D., Song E.J., Chang S., Yoo O.J., Yamazaki S., Lee C., and Takahashi J.S. 2005. A noncanonical E-box enhancer drives mouse Period 2 circadian oscillations in vivo. Proc. Natl. Acad. Sci. 102: 2608.

Yoshimura T., Yasuo S., Watanabe M., Iigo M., Yamamura T. Hirunagi K., and Ebihara S. 2003. Light-induced hormone conversion of $\mathrm{T} 4$ to $\mathrm{T} 3$ regulates photoperiodic response of gonads in birds. Nature 426: 178.

Young M.W. and Kay S.A. 2001. Time zones: A comparative genetics of circadian clocks. Nat. Rev. Genet. 2: 702

Zheng B., Larkin D.W., Albrecht U., Sun Z.S., Sage M., Eichele G., Lee C.C., and Bradley A. 1999. The mPer2 gene encodes a functional component of the mammalian circadian clock. Nature 400: 169.

Zheng B., Albrecht U., Kaasik K., Sage M., Lu W., Vaishnav S. Li Q., Sun Z.S., Eichele G., Bradley A., and Lee C.C. 2001. Nonredundant roles of the mPer1 and mPer2 genes in the mammalian circadian clock. Cell 105: 683.

Zylka M.J., Shearman L.P., Weaver D.R., and Reppert S.M. 1998. Three period homologs in mammals: Differential light responses in the suprachiasmatic circadian clock and oscillating transcripts outside of brain. Neuron 20: 1103 


\section{$\$_{\text {CSH\& }}^{\infty}$ Cold Spring Harbor Symposia SYMPOSIA}

\section{Systems Biology of Mammalian Circadian Clocks}

H. R. Ueda

Cold Spring Harb Symp Quant Biol 2007 72: 365-380

Access the most recent version at doi:10.1101/sqb.2007.72.047

References This article cites 77 articles, 24 of which can be accessed free at:

http://symposium.cshlp.org/content/72/365.full.html\#ref-list-1

License

Email Alerting Receive free email alerts when new articles cite this article - sign up in the box at the Service top right corner of the article or click here.

To subscribe to Cold Spring Harbor Symposia on Quantitative Biology go to: http://symposium.cshlp.org/subscriptions 